\title{
Research on Rosenbrock Function Optimization Problem Based on Improved Differential Evolution Algorithm
}

\author{
Jian Ma, Haiming $\mathrm{Li}^{*}$ \\ School of Computer Science and Technology, Shanghai University of Electric Power, Shanghai, China \\ Email: ${ }^{\star}$ zjxulhm@163.com
}

How to cite this paper: Ma, J. and Li, H.M. (2019) Research on Rosenbrock Function Optimization Problem Based on Improved Differential Evolution Algorithm. Journal of Computer and Communications, 7, 107-120. https://doi.org/10.4236/jcc.2019.711008

Received: September 20, 2019

Accepted: November 26, 2019

Published: November 29, 2019

Copyright (อ 2019 by author(s) and Scientific Research Publishing Inc. This work is licensed under the Creative Commons Attribution International License (CC BY 4.0).

http://creativecommons.org/licenses/by/4.0/

\section{(c) (i) Open Access}

\begin{abstract}
The Rosenbrock function optimization belongs to unconstrained optimization problems, and its global minimum value is located at the bottom of a smooth and narrow valley of the parabolic shape. It is very difficult to find the global minimum value of the function because of the little information provided for the optimization algorithm. According to the characteristics of the Rosenbrock function, this paper specifically proposed an improved differential evolution algorithm that adopts the self-adaptive scaling factor $F$ and crossover rate $C R$ with elimination mechanism, which can effectively avoid premature convergence of the algorithm and local optimum. This algorithm can also expand the search range at an early stage to find the global minimum of the Rosenbrock function. Many experimental results show that the algorithm has good performance of function optimization and provides a new idea for optimization problems similar to the Rosenbrock function for some problems of special fields.
\end{abstract}

\section{Keywords}

Differential Evolution, Rosenbrock Function, Self-Adaptive Mutation, Elimination Mechanism

\section{Introduction}

Differential evolution (DE) is a simple yet powerful search technique introduced by Storn and Price in 1995 [1]. It soon became a popular tool for solving global optimization problems because of several attractive features like having fewer control parameters, ease in programming, efficiency, etc. DE has a very special connection with artificial life, especially evolutionary algorithms. DE and par- 
ticle swarm optimization (PSO) are optimization algorithms based on collective intelligence theory [2]. They all use collective intelligence to optimize the result of searching, which comes from individual cooperation and competition within the group. However, compared to the evolutionary algorithm, DE retains the global search strategy based on population and uses real number coding, differential-based simple mutation operation and one-to-one competitive survival strategy, which reduces the complexity of genetic operations. At the same time, DE has special memory ability to track current search status dynamically to adjust the strategy of search. This capability indicates that the DE algorithm has strong global convergence ability and robustness without relying on the feature information of the problem, and the $\mathrm{DE}$ algorithm is suitable for solving optimization problems that cannot be solved by conventional mathematical programming methods in complex environments. Therefore, it is very academic and engineering value to conduct theoretical and applied research on DE [3], as an efficient parallel search algorithm.

In the minimization of the function, the phenomenon of "super valley" often occurs (at least in the local range) on the contour plane of the objective function. This shape is similar to the parabola with an upward opening. The global minimum value of the objective function is located at the bottom of the narrow valley. The optimization algorithm should have the ability to gradually approach the global minimum of the objective function along a narrow valley, and the Rosenbrock function of Rosenbrock, as a typical test function, was created for testing whether the optimization algorithm has this capability of searching the global minimum [4].

Many optimization algorithms now have a good optimization performance for most test functions, but much literature shows that many efficient algorithms and even many intelligent search algorithms are difficult to find global minimum values for the optimization of the Rosenbrock function, such as gradient descent, genetic algorithm (GA), particle swarm optimization (PSO) [5]. Specifically, for the gradient descent: when the objective function $f(x)$ is minimized by the gradient descent, the gradient of the function is the vector in the direction of maximum local growth of $f(x)$, and the opposite direction of the vector is the steepest downward direction. Because at point $x$, the negative gradient of $f(x)$ points to the direction in which $f(x)$ decreases the fastest for each component of $x$ and is orthogonal to the contour of $f(x)$ at $x$. Since the response surface of the Rosenbrock function has a curved and narrow valley, and the steepest descending direction of the point in the valley is almost perpendicular to the best direction leading to the minimum value of $f(x)$, the steepest drop around the curve in the valley. The gradient descent converges very slowly and does not converge at all even in a reasonable time. For GA, PSO and other intelligent search algorithms: In the early stage of the algorithm, these algorithms are easy to fall into local optimum on one side of the valley and cannot jump out because the global minimum value of the Rosenbrock function is at the bottom of the valley. 
This paper proposes an improved differential evolution algorithm with selfadaptive mutation and control parameters to solve this kind of problem and optimize the characteristic design of the Rosenbrock function. In this algorithm, the scaling factor $F$ can be adaptively changed by the previous learning experience, and the level of the crossover rate $C R$ depends on the fitness value of the individual. Experiments show that the improved DE algorithm has better convergence speed and accuracy than other types of improved schemes.

\section{Basic Differential Evolution Algorithm}

Starting from a population of randomly initialized solutions, the DE algorithm employs simple mutation and crossover operators to generate new candidate solutions and utilizes a one-to-one competition scheme to deterministically decide whether the offspring will replace their parents in the next generation. The steps of the standard DE algorithm are as follows:

1) Population initialization

The matrix $X$ defining $N \times D$ is used to store the data of the current population, where $D$ is the dimension of the individual in the population, $N$ is the size of the population. At first, it randomly generates $N \times D$ data obeying the uniform distribution law and satisfying the specific constraint conditions. Put them into the matrix $X$ to form the initial population

$$
X(0)=\left\{X_{1}(0), X_{2}(0), \cdots, X_{N}(0)\right\} .
$$

2) Variation operation

Let the population size be $N$, the current evolutionary individual is represented by $X_{i}(t) \quad(i=1,2, \cdots, N)$ and $t$ is the current number of evolutions. Randomly select three different individuals from the current evolutionary group and meet the conditions $r_{1}, r_{2}, r_{3} \in\{1,2, \cdots, N\}$. Individual $X_{r 2}(t), X_{r 3}(t)$ and difference $X_{r 2}(t)-X_{r 3}(t)$ are regarded as the disturbance factors of $X_{r 1}(t)$. Add the factors and $X_{r 1}(t)$ to obtain the current mutant individual $V_{i}(t)$, as shown in Equation (1):

$$
V_{i}(t)=X_{r i}(t)+F\left(X_{r 2}(t)-X_{r 3}(t)\right)
$$

where $F$ is the scaling factor that controls the disturbance factor. Individuals in a group are composed of $D$ components, then variant individuals $V_{i}(t)$ also composed of $D$ components, so $V_{i}(t)=\left(v_{r 1}(t), v_{r 2}(t), \cdots, v_{i D}(t)\right)$.

3) Crossover operation

Generate random integers $r_{4} \in\{1,2, \cdots, D\}$. The discrete variant individual $V_{i}(t)$ crosses the current evolutionary individual $X_{i}(t)$ to obtain an intermediate test individual $U_{i}(t)$, which will compete with the $X_{i}(t)$ for the selection operation and $U_{i}(t)$ consists of $D$ component, $U_{i}(t)=\left(u_{i 1}(t), u_{i 2}(t), \cdots, u_{i D}(t)\right)$. The $t$ th component (the chromosome in the individual) is as shown in Equation (2):

$$
u_{i j}(t)\left\{\begin{array}{l}
v_{i j}(t) \operatorname{randj}(0,1) \leq C R \text { or } j=r_{4} \\
v_{i j}(t) \operatorname{randj}(0,1)>C R \text { or } j \neq r_{4}
\end{array}\right.
$$


$r_{4} \in\{1,2, \cdots, D\}$ and randj $(0,1)$ represents a uniformly distributed random number obeying $(0,1)$, which can guarantee that the individual is mutated $V_{i}(t)$ at least one dimension component exists in the test individual $U_{i}(t)$ to ensure the effectiveness of the mutation operation. $C R \in[0,1]$ is the probability of crossover. The lower the probability of $C R$, the similarity of the intermediate test individual $U_{i}(t)$ for current evolutionary individual $X_{i}(t)$ is greater. This will help ensure the diversity of the population and the global optimization of the algorithm.

4) Selection operation

In the case of seeking a minimum solution, the smaller the fitness value, the better. If the fitness value of $U_{i}(t)$ is less than the fitness value, the intermediate individual $U_{i}(t)$ will replace the current individual $X_{i}(t)$ with the new individual $X_{i}(t+1)$. Otherwise, the current evolutionary individual $X_{i}(t)$ will inherit directly into the next generation. If the individual fitness value is $f\left(X_{i}(t)\right)$, the selection operation can be expressed as follows (3):

$$
X_{i}(t+1)=\left\{\begin{array}{l}
U_{i}(t) \text { if } f\left(U_{i}(t)\right) \leq f\left(X_{i}(t)\right) \\
X_{i}(t) \text { if } f\left(U_{i}(t)\right)>f\left(X_{i}(t)\right)
\end{array}\right.
$$

5) Evaluation

In summary, the DE algorithm has the following advantages:

a) The algorithm is generic and does not depend on the problem information;

b) The algorithm principle is simple and easy to implement;

c) Group search, with the ability to memorize the optimal solution of individuals;

d) Collaborative search, with the ability to further search using individual local information and group global information guidance algorithms;

e) It is easy to mix with other algorithms to construct an algorithm with better performance.

The search performance of the DE algorithm depends on the balance between the global exploration of the algorithm and the local development capability, which depends to a large extent on the selection of the control parameters of the algorithm, including population size, scaling factor and crossover probability. Compared with other evolutionary algorithms, the $\mathrm{DE}$ algorithm requires fewer parameters to adjust. It is necessary to select reasonable parameters for the guidance of selection.

\section{The Analysis of the Rosenbrock Function}

The Rosenbrock function is a classic function in unconstrained optimization theory and method. It is an important tool to measure the advantages and disadvantages of unconstrained algorithms. In the field of numerical optimization, this function was proposed by Howard H. Rosenbrock in 1960. The function is a typical non-convex function, mainly used to optimize the performance test of the algorithm, and is known as the Rosenbrock valley function or the Rosenbrock banana function. 


\subsection{Definition of the Rosenbrock Function}

The Rosenbrock function is defined as follows:

$$
f(x, y)=(1-x)^{2}+100\left(y-x^{2}\right)^{2}
$$

Each contour of the Rosenbrock function is roughly parabolic, whose global minimum is also in a parabolic valley (banana-type valley). It is easy to find this valley but quite difficult to find the minimum of the whole domain because the value in the valley does not change much.

The global minimum of the function is at $(x, y)=(1,1)$ and the value is $f(x, y)$ $=0$. Sometimes the coefficient of the second term (100 in the above formula) is different, but it does not affect the position of the global minimum. This paper mainly focuses on the Rosenbrock function in the two-dimensional case.

The three-dimensional map of the two variables of the Rosenbrock function is shown in Figure 1.

The more complex form of Rosenbrock function under multivariate [6] as follows:

$$
f(x)=\sum_{i=1}^{N-1}\left[\left(1-x_{i}\right)^{2}+100\left(x_{i+1}-x_{i}^{2}\right)^{2}\right], \forall x \in R^{N}
$$

It can be proved that when $N=3$, this form of Rosenbrock function has only a minimum value, and the position is $(1,1,1)$, in $4 \leq N \leq 7$, there are only two minimum values. When all variables are 1 , there is a global minimum $\left(x_{1}, x_{2}, \cdots, x_{N}=(-1,1, \cdots, 1)\right)$. There is a local minimum nearby. This result is obtained after the gradient of the function is zero. The Rosenbrock function is an unconstrained function optimization problem, which exhibits the characteristics of a multimodal function with a dimension greater than 3 and a unimodal indivisible function with other dimensions.

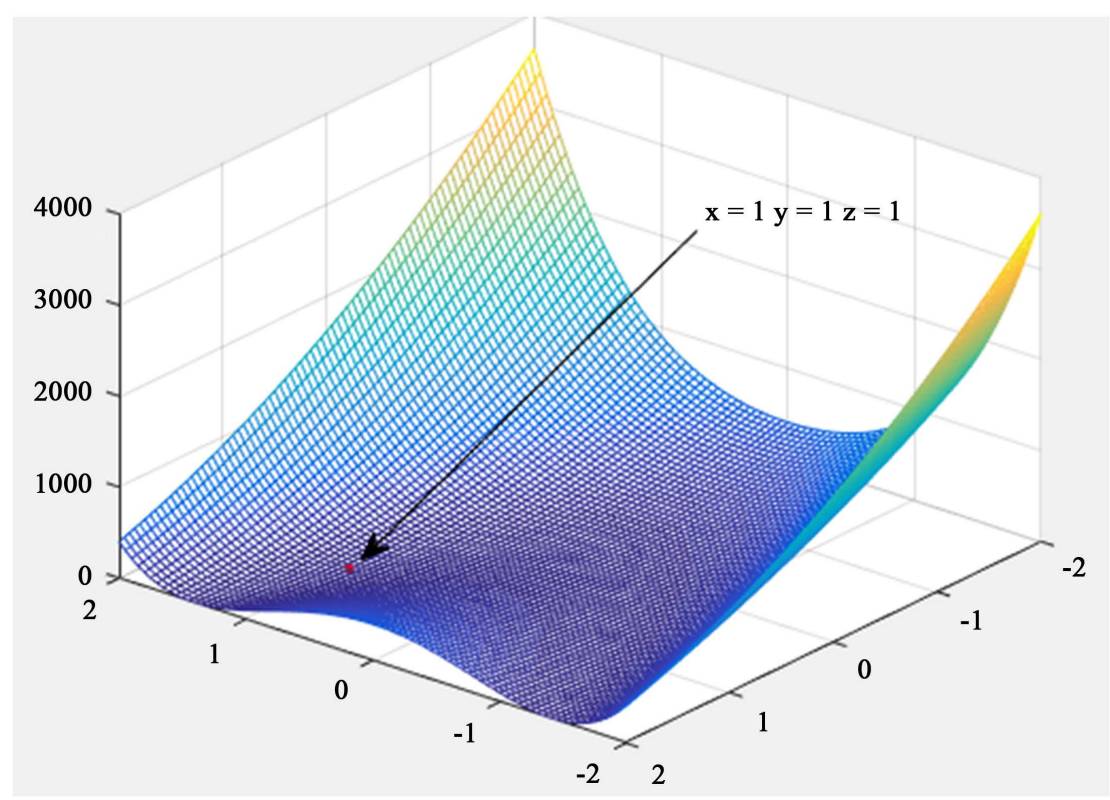

Figure 1.3D graph of Rosenbrock function. 


\subsection{The Analysis of the Rosenbrock Function}

As you can see in Figure 1, the global minimum of the Rosenbrock function is at the bottom of a valley with a smooth and narrow parabola shape. It is difficult for general optimization algorithms to distinguish the search direction because of the less search information provided. It becomes difficult to find the global minimum value and easy to fall into a local optimum on one side of the valley. In the final search phase, these algorithms cannot jump out when the algorithm optimizes the Rosenbrock function.

This paper proposes a further improvement of the original differential evolution algorithm, which combines the multi-strategy crossover operation and the self-adaptive dynamic adjustment algorithm parameters so that the algorithm takes into account both global search and local search. The new algorithm improves the convergence speed and accuracy of the optimization on the Rosenbrock function. The test results below verify the superiority of the algorithm.

\section{Improved Differential Evolution Algorithm}

\subsection{Scaling Factor $F$}

By literature [7], it can be seen that when the scaling factor $F$ is between $[0.5,1]$, the algorithm obtains better results. When $F<0.5$ or $F>1$, the quality of the solution obtained by the algorithm is not high. And the literature shows that the average optimal value is ideal for almost all test functions at $F=0.5$. Therefore, we take the benchmark value $F_{\min }=0.5, F_{\max }=1$ in the paper.

It can be known from Equation (1) that the value of $F$ directly affects the convergence speed and convergence of the algorithm: the scaling factor $F$ controls the amplitude of the difference vector, and its value also affects the convergence and convergence speed. When $F$ is small, the convergence speed is faster, but if it is too small, it tends to converge to the non-optimal solution; if $F$ is large, it is conducive to convergence to the optimal solution, but the convergence speed is slower.

We need to maintain the diversity of the population in the initial stage of the search, and we should get as many individuals as possible globally optimal when doing a global search. We should also strengthen the ability of local search in the later stage of the search to improve the accuracy of the algorithm.

Therefore, we take such a measure for the value of $F$ : The previous $F$ takes a larger value to increase the mutation rate and ensure the ability of global search. And we reduce the $F$ as the number of iterations increases, which can improve the ability of local search. We can propose an improved scheme of self-adaptive F: By literature [8], a linearly decreasing $F=F_{\max }-\frac{t\left(F_{\max }-F_{\min }\right)}{T}\left(F_{\max }\right.$ and $F_{\min }$ are the maximum and minimum values of $F, t$ is the current number of iterations, and $T$ is the maximum number of iterations) was proposed instead of the traditional fixed value $F$. By literature [9], a nonlinearly decreasing $F$ strategy 
was proposed: $F=F_{\max }-\left(F_{\max }-F_{\min }\right)\left(\frac{g}{G_{\max }}\right)^{2}$ ( $g$ is the current number of iterations, $G_{\max }$ is the maximum number of iterations), the function of the strategy is similar to a parabola with an opening down.

This paper uses an exponential declining strategy that is flatter and smoother than the first two.

$$
F=F_{0} * 2^{\mathrm{e}^{\left(1-\frac{T}{T+1-t}\right)}}
$$

In the above formula, $F_{0}$ is equivalent to $F_{\min }, t$ and $T$ are the current number of iterations and the maximum number of iterations, respectively. At the beginning of the algorithm, the self-adaptive mutation operator $F$ is $2 F_{0}$, which means that the individual diversity can be maintained at the initial stage because of a large mutation rate; as the algorithm progresses, the mutation rate decreases gradually and will be close to $F_{0}$ at the end of the algorithm, thus avoiding the destruction of the optimal solution.

\subsection{Crossover Rate $C R$}

The size of the crossover rate $C R$ has a great influence on the convergence and convergence speed of the algorithm. It can be seen from Equation (2) that the larger the value of $C R$, the more $V_{i}(t)$ contributes to $U_{i}(t)$, which means that there will be more variant individuals in the crossover operation. This trend is conducive to opening up new space and accelerating convergence.

However, the mutated individuals tend to be the same at a later stage (the self-adaptive values of the mutated individuals tend to be the same), which is not conducive to maintaining diversity, so it is easy to fall into the local optimal solution, and the stability of this kind of algorithm is poor; the smaller the value of $C R$, the more $X_{i}(t)$ contributes to $U_{i}(t)$. In this way, the ability of the algorithm to develop new space is weakened, and the convergence speed is relatively slow, but it is beneficial to maintain the diversity of the population (retaining the original individual characteristics), and thus the algorithm has a higher success rate. Therefore, we should choose to preserve the diversity of the population more stable in the early stage, and develop slowly, gradually increasing the $C R$ to accelerate the accurate convergence in the later stage, and not easily fall into the local optimum.

By literature [10], four improvement strategies were proposed to improve the original fixed value $C R$. As a result, the optimization performance of the open-up parabola form is the best. The parameters are as follows:

$$
C R=\left(C R_{\max }-C R_{\min }\right)\left(\frac{g}{G_{\max }}\right)^{2}+C R_{\min }
$$

$C R_{\max }, C R_{\min }, g, G_{\max }$ are the maximum and minimum values of $C R$, the current iteration number and the maximum number of iterations, respectively.

In this paper, we consider that the differential evolution algorithm only eva- 
luates the fitness of the function in the final selection. Is it possible to filter the mutated individuals in advance at the crossover operation, which provides more opportunities for excellent individuals to be selected at the crossover operation; while disadvantaged individuals are given a lower probability and constantly being eliminated. Therefore, this paper proposes a kind of elimination mechanism based on the fitness of individuals (taking the minimum value as an example):

$$
C R= \begin{cases}C R_{\min }+\left(C R_{\max }-C R_{\min }\right) * \frac{f_{i}-f_{a v}}{f_{\text {max }}-f_{a v}}, & \text { if } f_{i}<f_{a v} \\ C R_{\min }, & \text { otherwise }\end{cases}
$$

Set up a $C R$ with maximum and minimum values called $C R_{\max }, C R_{\min }$. At the same time, the average value of all fitness functions is calculated at each iteration $f_{a v}$ and search for maximum fitness $f_{\max }$. We use a mechanism for self-adaptive $C R$ regulation at the crossover operation:

Suppose we are looking for a global minimum. If the average value of the population $f_{a v}$ is lower than the current fitness value of the individual $f_{i}$, we can treat this individual as a dominant individual. The crossover rate $C R$ for the individual will increase with the degree of approximation to the maximum fitness. If the average value of the population $f_{a v}$ is higher than the current fitness value of the individual $f_{i}$, we can treat this individual as an inferior individual. The crossover rate $C R$ for the individual will be set to the lowest $C R_{\min }$. Therefore, the dominant individual will be continued, and the inferior individual will gradually decrease. The method also follows the principle that the $C R$ increases with the number of iterations.

As the algorithm reaches the end, the better fitness value of the function will be retained, the $C R$ will be closer to the minimum value of the current iteration, which will help accelerate the convergence of the algorithm and improve the accuracy. The following Figure 2 shows the flow chart of the improved differential evolution algorithm.

We can call this strategy of simultaneously improved $F-C R$ parameters as IEDE (Index-Elimination Differential Evolution Algorithm).

\section{Experimental Results and Performance Analysis}

\subsection{Parameter Settings}

In the experiment, we select the standard DE algorithm and the improved algorithm for performance test comparison. The parameters selected in the experiment are as follows: the population size is $N=5 D-10 D$ and $N=50$ in this paper. $C R=0.5, F_{0}=0.5, f_{\min }=0.5, f_{\max }=1, C R_{\min }=0.3, C R_{\max }=0.9$ in the improved algorithm. The maximum number of iterations is 200 under the twodimensional variable of the Rosenbrock function. The accuracy of the experimental test is set to $1 \mathrm{e}-4$, which means that if the result reaches a difference of 1e -4 from the target value and tends to be constant, then we can conclude that the iteration is over. 


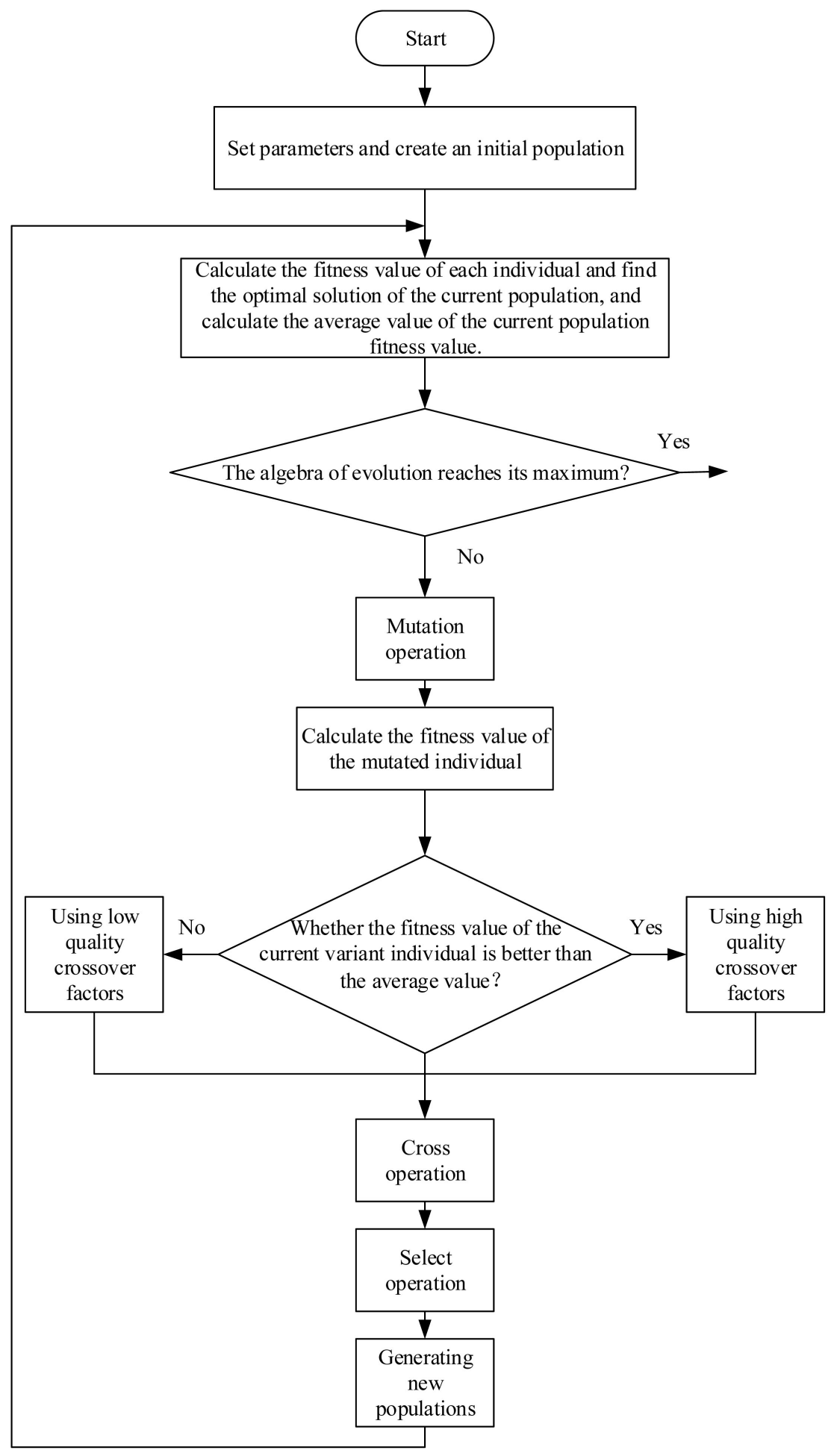

Figure 2. Flow chart for the improved DE algorithm.

\subsection{Experimental Results and Performance Analysis}

Take the standard differential evolution algorithm and the improved algorithm (IEDE) as an example, and use python to make graphs and compare them. As shown in Figure 3 below. 
MIN: $1.4186545766642806 \mathrm{e}-22$

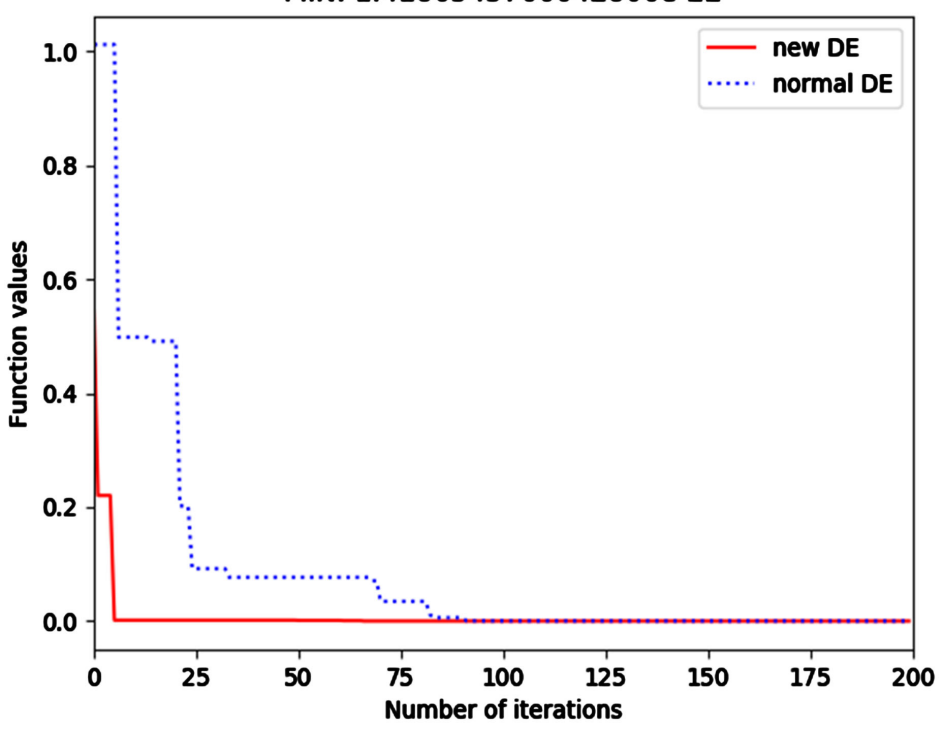

Figure 3. Performance comparison of the two algorithms.

It can be seen from Figure 3 that the fitness of the newly improved differential evolution algorithm is closer to the optimal value $(f=0)$ than the standard differential evolution algorithm, and the best solution of this iteration in the figure is $\left(x_{0}, x_{1}\right)=(0.9999999999935465,0.9999999999860919)$, the final function value is $1.4186545766642806 \mathrm{e}-22$.

At the same time, we perform each algorithm of the other literature20 times and record the number of times it takes to reach the number of stable iterations to verify the superiority of the newly improved differential evolution algorithm. The results are shown in Table 1 and Table 2.

It can be seen from Table 2 that in the two improved schemes of the crossover rate $C R$, the $C R$-elimination mechanism is obviously superior to the $C R$ parabolic line, and it has achieved excellent results. It can be seen from the comparison of the standard deviation that the standard deviation corresponding to the $C R$-elimination mechanism is the smallest, indicating that the scheme has high repeatability.

Among three schemes of the scaling factor $F$, the effect of the $F$-parameter in the exponential form is better than that of the linear and parabolic methods (we adopt the control variable method: The $C R$ is consistent with the parameters in the standard DE algorithm when we test $F$ ), but the iteration cost is more than the standard DE algorithm. This is because the self-adaptive characteristics of the $F$ parameter are not reflected when the dimension is low, and the global optimum can be easily found without changing the $F$.

Therefore, the second round of testing is carried out to increase the dimension and increase the maximum number of iterations. In all the schemes with improved $F$ parameters, the $C R$ factor uniformly adopts the $C R$-elimination mechanism proposed in this paper. This time, we take a maximum number of iterations of 1000 and a dimension of 5 . Considering that only $D=5$ is taken, the 
Table 1. The number of iterations required for each scheme to stabilize.

\begin{tabular}{|c|c|c|c|c|c|c|}
\hline $\begin{array}{l}\text { Experiment } \\
\text { number }\end{array}$ & $\begin{array}{l}\text { Normal } \\
\text { DE }\end{array}$ & $\begin{array}{c}F \text {-linear } \\
{[8]}\end{array}$ & $\begin{array}{c}F \text {-parabola } \\
{[9]}\end{array}$ & $F$-index & $\begin{array}{c}C R \text {-parabola } \\
{[10]}\end{array}$ & $C R$-elimination \\
\hline 1 & 88 & 103 & 126 & 101 & 146 & 34 \\
\hline 2 & 94 & 166 & 143 & 132 & 150 & 30 \\
\hline 3 & 89 & 98 & 193 & 119 & 132 & 44 \\
\hline 4 & 81 & 142 & 161 & 115 & 135 & 26 \\
\hline 5 & 87 & 140 & 160 & 132 & 93 & 43 \\
\hline 6 & 88 & 72 & 141 & 110 & 113 & 28 \\
\hline 7 & 125 & 109 & 144 & 111 & 112 & 27 \\
\hline 8 & 68 & 123 & 172 & 127 & 131 & 38 \\
\hline 9 & 86 & 112 & 131 & 120 & 135 & 26 \\
\hline 10 & 95 & 115 & 157 & 129 & 118 & 20 \\
\hline 11 & 93 & 105 & 145 & 133 & 117 & 46 \\
\hline 12 & 86 & 129 & 150 & 118 & 145 & 50 \\
\hline 13 & 113 & 127 & 133 & 106 & 146 & 50 \\
\hline 14 & 96 & 162 & 132 & 109 & 152 & 50 \\
\hline 15 & 77 & 132 & 129 & 117 & 139 & 33 \\
\hline 16 & 75 & 149 & 152 & 85 & 139 & 38 \\
\hline 17 & 89 & 133 & 98 & 144 & 166 & 49 \\
\hline 18 & 101 & 158 & 157 & 155 & 166 & 49 \\
\hline 19 & 97 & 139 & 134 & 113 & 151 & 42 \\
\hline 20 & 90 & 140 & 160 & 136 & 173 & 35 \\
\hline
\end{tabular}

Table 2. Experimental results under different improvement schemes.

\begin{tabular}{ccccc}
\hline Algorithm & $\begin{array}{c}\text { The maximum } \\
\text { number of iterations }\end{array}$ & $\begin{array}{c}\text { The average } \\
\text { number of iterations }\end{array}$ & $\begin{array}{c}\text { Minimum number } \\
\text { of iterations }\end{array}$ & $\begin{array}{c}\text { Standard } \\
\text { deviation }\end{array}$ \\
\hline Normal DE & 125 & 90.9 & 68 & 12.57357 \\
F-linear [8] & 166 & 127.7 & 72 & 23.60664 \\
F-parabola [9] & 193 & 145.9 & 98 & 19.94967 \\
F-index & 155 & 120.6 & 85 & 15.90895 \\
CR-parabola [10] & 173 & 137.95 & 93 & 20.19764 \\
CR-elimination & 50 & 31 & 20 & 9.62945 \\
\hline
\end{tabular}

Normal DE refers to the standard DE algorithm without any changes; $F$-linear refers to changing $F$ to self-adaptive $F=F_{\max }-\frac{t\left(F_{\max }-F_{\min }\right)}{T} ; F$-parabola refers to changing $F$ to self-adaptive $F=F_{\max }-\left(F_{\max }-F_{\min }\right)\left(\frac{g}{G_{\max }}\right)^{2} ; F$-index refers to changing $F$ to self-adaptive $F=F_{0} * 2^{\left(\frac{1}{\left.1-\frac{T}{T+1-t}\right)} ; C R \text {-parabola }\right.}$ refers to changing $C R$ to self-adaptive $C R=\left(C R_{\max }-C R_{\min }\right)\left(\frac{g}{G_{\max }}\right)^{2}+C R_{\min } ; C R$-elimination refers to changing $C R$ to self-adaptive $C R=\left\{\begin{array}{ll}C R_{\min }+\left(C R_{\max }-C R_{\min }\right) * \frac{f_{i}-f_{a v}}{f_{\max }-f_{a v}}, & \text { if } f_{i}>f_{a v} \\ C R_{\min }, & \text { otherwise }\end{array}\right.$. 
third round of testing is performed, taking $D=10$, and the maximum number of iterations is 3000 . We perform 20 times for each scheme and calculate the mean and standard deviation.

The experimental results are shown in Table 3, Table 4 below.

It can be seen from the table that the standard DE algorithm cannot adapt to the computational difficulty brought by high-dimensional with the improvement of the dimension. At this time, the self-adaptive change advantage of $F$ is reflected. The original standard algorithm cannot meet the requirements of highdimension, so we draw a graph comparison in these three schemes. As shown in Figure 4 and Figure 5 below.

MIN: 3.95042235305091e-21

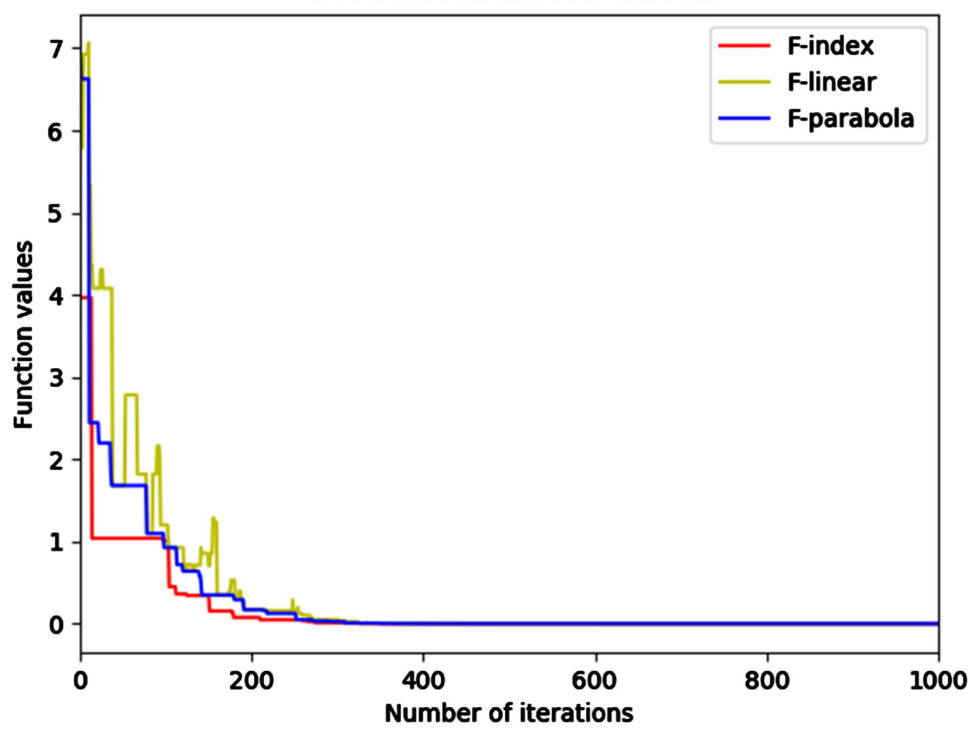

Figure 4. Changing curves of fitness value under three schemes $(D=5)$.

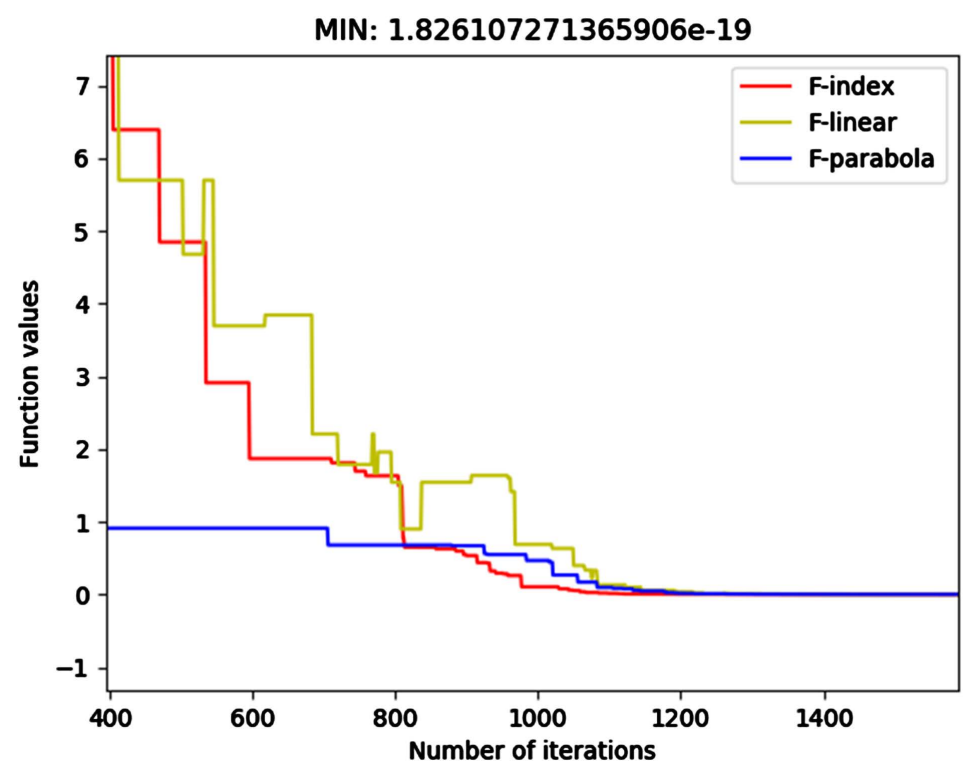

Figure 5. Changing curves of fitness value under three schemes $(D=10)$. 
Table 3. Experimental results at $D=5$.

\begin{tabular}{ccc}
\hline Algorithm & Mean & Standard deviation \\
\hline Normal DE & 852 & 60.25529 \\
F-index & 373 & 30.12806 \\
F-parabola [9] & 400 & 49.33322 \\
F-linear [8] & 470 & 49.09888 \\
\hline
\end{tabular}

Table 4. Experimental results at $D=10$.

\begin{tabular}{ccc}
\hline Algorithm & Mean & Standard deviation \\
\hline Normal DE & 2456 & 195.3286 \\
$F$-index & 1239 & 181.0064 \\
$F$-parabola [9] & 1440 & 173.6327 \\
$F$-linear [8] & 1478 & 203.6981 \\
\hline
\end{tabular}

We can see from Figure 4 and Figure 5 that in the three improved schemes, the improved DE algorithm in the $F$-index form proposed in this paper takes the least number of times to achieve stable iteration. When $D=5$, the efficiency of the IEDE is increased by $128.4 \%$ compared with the standard DE algorithm, and the standard deviation is the lowest among the four schemes, which means that the scheme of the IEDE is the most efficient and stable, and the repeatability of the IEDE is higher than other schemes. It can be seen that the improved DE algorithm (IEDE) using the $F$-index- $C R$ elimination mechanism can effectively improve the operation speed of the original algorithm.

\section{Conclusion}

Many optimization algorithms are difficult to find its global minimum because of the fact that the global minimum of the Rosenbrock function is at the bottom of a narrow valley. This paper proposes a new improved DE algorithm (IEDE). The improved algorithm studies and analyzes various improvement schemes and proposes a new exponential strategy of a self-adaptive scaling factor in the mutation, and adopts the individual elimination mechanism which can adjust the crossover rate by distinguishing between superior individuals and inferior individuals in the crossover. Experimental studies show that the IEDE algorithm has very good performance for the optimization of the Rosenbrock function and further improves the convergence speed and accuracy of the algorithm and it can maintain certain stability even in high dimensions. At the same time, the Rosenbrock function also proves that the IEDE algorithm is a good performance optimization algorithm. The next research work is how to further improve the efficiency of the IEDE algorithm and to extend it to all optimization problems. 


\section{Conflicts of Interest}

The authors declare no conflicts of interest regarding the publication of this paper.

\section{References}

[1] Price, K. (1996) Differential Evolution: A Fast and Simple Numerical Optimizer. Biennial Conference of the North American Fuzzy Information Processing Sociey, Berkeley, 19-22 June 1996, 524-527.

[2] Lu, X., Ke, T., Sendhoff, B. and Yao, X. (2014) A New Self-Adaptation Scheme for Differential Evolution. Neurocomputing, 146, 2-16. https://doi.org/10.1016/j.neucom.2014.04.071

[3] Liu, B., Wang, L. and Jin, Y. (2007) Advances in Differential Evolution. Kongzhi yu Juecel Control and Decision, 22, 721-729.

[4] Rosenbrock, H.H. (1960) An Automatic Method for Finding the Greatest or Least Value of a Function. The Computer Journal, 3, 175-184. https://doi.org/10.1093/comjnl/3.3.175

[5] Kennedy, J. and Eberhart, R. (1995) Particle Swarm Optimization. Proceedings of IEEE International Conference on Neural Networks, Perth, Australia, 27 November-1 December 1995, 1942-1948.

[6] Yang, X.S. and Deb, S. (2010) Engineering Optimization by Cuckoo Search. International Journal of Mathematical Modelling and Numerical Optimisation, 1, 330-343. https://doi.org/10.1504/IJMMNO.2010.035430

[7] Storn, R. (1996) On the Usage of Differential Evolution for Function Optimization. Biennial Conference of the North American Fuzzy Information Processing Sociey, Berkeley, 19-22 June 1996, 519-523.

[8] Gao, Y.L. and Liu, J.F. (2008) Adaptive Differential Evolution Algorithm. Journal of Hebei University of Engineering, 8, 11-14.

[9] Xiao S.-J. and Zhu, X.-F. (2009) A Modified Fast and Highly Efficient Differential Evolution Algorithm. Journal of Hefei University of Technology (Natural Science), 32, 1700-1703.

[10] Deng, Z.-X. and Liu, X.-J. (2008) Study on Strategy of Increasing Cross Rate in Differential Evolution Algorithm. Computer Engineering \& Applications, 44, 33-36. 